\title{
MULTIDIMENSIONAL COPULA MODELS FOR PARALLEL DEVELOPMENT OF THE US BOND MARKET INDICES
}

\author{
Jozef Komorník - Magdaléna KomorníKovÁ - \\ - Tomáš Bacigál - CuONG NGuYen
}

\begin{abstract}
Stock and bond markets co-movements have been studied by many researchers. The object of our investigation is the development of three U.S. investment grade corporate bond indices. We concluded that the optimal 3D as well as partial pairwise 2D models are in the Student class with 2 degrees of freedom (and thus very heavy tails) and exhibit very high values of tail dependence coefficients. Hence the considered bond indices do not represent suitable components of a well-diversified investment portfolio. On the other hand, they could make good candidates for underlying assets of derivative instruments.
\end{abstract}

\section{Introduction}

In this paper, we apply 2- and 3-dimensional copula models to the triple of time series of returns of indices of US financial markets (using daily data from Bloomberg). The triple of indices (US CBI) contains the Bank of America Merrill Lynch US Corporate Bond Index (ML), the Barclays US Corporate \& Investment Grade Index (BAR), Dow Jones Corporate Bond Index (DJ) from the time period from January 1997 to May 2014. Our results show high values of Kendalls correlation coefficients as well as tail dependencies between all couples of this triple of indices.

The paper is organized as follows. The second section is devoted to a brief overview of the theory of copulas. In the third section we present the utilized methodology of copula fitting to two- and three-dimensional time series. The fourth section contains application to real data modeling. Finally, some conclusions are presented.

(C) 2017 Mathematical Institute, Slovak Academy of Sciences. 2010 Mathematics Subject Classification: 62H20, 62H86, 62P20, $91 \mathrm{G} 70$.

Keywords: bond market indices, Copulas, Cramer-von Mises test statistics, GoF test, Vuong and Clarke tests.

The support of the grants APVV-0013-14 and VEGA 1/0420/15 is kindly announced. 


\section{J. KOMORNÍK - M. KOMORNÍKOVÁ - T. BACIGÁL - C. NGUYEN}

\section{Copulas}

Copula represents a multivariate distribution that captures the dependence structure among random variables. It is a great tool for building flexible multivariate stochastic models. Copula offers the choice of an appropriate model for the dependence between random variables independently from the selection of marginal distributions. This concept was introduced in the late 50's and became popular in several fields beyond statistics and probability theory, such as finance, actuarial science, fuzzy set theory, hydrology, civil engineering, etc.

Due to 11

$$
F\left(x_{1}, \ldots, x_{n}\right)=C\left[F_{1}\left(x_{1}\right), \ldots, F_{n}\left(x_{n}\right)\right],
$$

where $F$ is joint cumulative distribution function of random vector $\left(X_{1}, \ldots, X_{n}\right)$, $F_{i}, i=1, \ldots, n$ is marginal cumulative distribution function of $X_{i}$, and $C$ : $[0,1]^{n} \rightarrow[0,1]$ is a copula which is $n$-increasing function with 1 as neutral element and 0 as annihilator, see, e.g., [8].

Besides three fundamental copulas

$$
\begin{gathered}
M\left(x_{1}, \ldots, x_{n}\right)=\min \left\{x_{1}, \ldots, x_{n}\right\}, \quad W\left(x_{1}, x_{2}\right)=\max \left\{x_{1}+x_{2}-1,0\right\}, \\
\Pi\left(x_{1}, \ldots, x_{n}\right)=\prod_{i=1}^{n} x_{i}
\end{gathered}
$$

which model perfect positive dependence, perfect negative dependence (not applicable for $n>2$ ) and independence, respectively, there exist numerous parametric classes, such as Archimedean, Extreme-Value and elliptical copulas. Within the last there belong such important parametric families as Gaussian copulas

$$
C_{G}\left(x_{1}, \ldots, x_{n}\right)=\Phi\left[\Phi_{1}^{-1}\left(x_{1}\right), \ldots, \Phi_{n}^{-1}\left(x_{n}\right)\right]
$$

and Student t-copulas

$$
C_{t}\left(x_{1}, \ldots, x_{n}\right)=t\left[t_{1}^{-1}\left(x_{1}\right), \ldots, t_{n}^{-1}\left(x_{n}\right)\right]
$$

(where $\Phi$ and $t$ are joint distribution functions of multivariate normal and Student $t$ distributions, similarly $\Phi_{i}^{-1}$ and $t_{i}^{-1}$ are univariate quantile functions related to $X_{i}$ ), able to flexibly describe dependence in multidimensional random vector.

The Archimedean class [5]

$$
C_{A}\left(x_{1}, \ldots, x_{n}\right)=\phi^{(-1)}\left[\phi\left(x_{1}\right)+\cdots+\phi\left(x_{n}\right)\right]
$$

(with generator $\phi:[0,1] \rightarrow[0, \infty]$ and its pseudo-inverse $\phi^{(-1)}$ ) is much easier to handle, yet it is reasonably useful only in two-dimensional case. 
Tail dependencies are functions that describe the dependence structure of multi-dimensional distributions in the tail and are defined (for bivariate case) as follows [8].

Let $X_{1}$ and $X_{2}$ be continuous random variables with distributions functions $F_{1}$ and $F_{2}$ and with copula $C$, then the lower tail dependence coefficient is defined by

$$
\lambda_{L}=\lim _{u \rightarrow 0^{+}} \operatorname{Pr}\left(X_{1} \leq F_{1}^{-1}(u) \mid X_{2} \leq F_{2}^{-1}(u)\right)=\lim _{u \rightarrow 0^{+}} \frac{C(u, u)}{u},
$$

and the upper tail dependence coefficient by

$$
\lambda_{U}=\lim _{u \rightarrow 1^{-}} \operatorname{Pr}\left(X_{1}>F_{1}^{-1}(u) \mid X_{2}>F_{2}^{-1}(u)\right)=\lim _{u \rightarrow 1^{-}} \frac{1-2 u+C(u, u)}{1-u} .
$$

(provided that the above limits exist).

Note that in the above formulas (4) and (5), the role of $X_{1}$ and $X_{2}$ are exchangeable.

Analogically for a $n$-dimensional copula $C\left(x_{1}, \ldots, x_{n}\right)$ we define [3]

and

$$
\lambda_{L, i}=\lim _{u \rightarrow 0^{+}} \operatorname{Pr}\left(X_{i} \leq F_{i}^{-1}(u) \mid F_{j}^{-1}(u) \leq u \text { for all } j \neq i\right)
$$

$$
\lambda_{U, i}=\lim _{u \rightarrow 1^{-}} \operatorname{Pr}\left(X_{i} \geq F_{i}^{-1}(u) \mid F_{j}^{-1}(u) \geq u \text { for all } j \neq i\right) .
$$

Since the role of the variables for Archimedean copulas is exchangeable, the above formulas (6) and (7) of $\lambda_{L, i}$ and $\lambda_{U, i}$ do not depend on $i$ for them.

Archimedean copulas can capture different tail dependencies, i.e., $\lambda_{L} \neq \lambda_{U}$. However, their exchangeability property can cause problems for their utilization for modeling data in higher dimensions. The Gaussian copulas do not have lower and upper tail dependencies. The $t$ copula has upper tail dependencies $\lambda_{U, i}$ (and because of radial symmetry) equal lower tail dependence $\lambda_{L, i}$ for $i=1, \ldots, n$.

\section{Fitting of copulas}

Given $m$ observations $\left\{X_{j, i}\right\}_{i=1, \ldots, m}$ of $\mathrm{j}$-th random variable $X_{j}$, the parameters $\theta$ of all copulas under consideration were estimated by maximizing the likelihood function

$$
L(\theta)=\sum_{i=1}^{m} c_{\theta}\left(U_{1, i}, U_{2, i}, U_{3, i}\right),
$$

where $c_{\theta}$ denotes density of a parametric copula family $C_{\theta}$, and

$$
U_{j, i}=\frac{1}{m+1} \sum_{k=1}^{m} \mathbf{1}\left(X_{j, k} \leq X_{j, i}\right), \quad i=1, \ldots, m
$$




\section{J. KOMORNÍK - M. KOMORNÍKOVÁ - T. BACIGÁL - C. NGUYEN}

are so-called pseudo-observations. Goodness-of-fit was performed by a test proposed by [6] and based on empirical copula process using Cramer-von Misses test statistic

$$
S_{C M}=\sum_{i=1}^{m}\left[C_{\theta}\left(U_{1, i}, U_{2, i}, U_{3, i}\right)-C_{m}\left(U_{1, i}, U_{2, i}, U_{3, i}\right)\right]^{2}
$$

with empirical copula

$$
C_{m}(\mathbf{x})=\frac{1}{m} \sum_{i=1}^{m} \prod_{j=1}^{3} \mathbf{1}\left(X_{j, i} \leq x_{j}\right)
$$

and indicator function $\mathbf{1}(A)=1$ whenever $A$ is true, otherwise $\mathbf{1}(A)=0$.

For selection of the optimal copula for a given couple, we utilized the scoring criteria based on Vuong and Clarke Tests [1].

The test proposed by Clarke (2007) 2 allows to compare non-nested models with copulas having densities $c_{1}$ and $c_{2}$ with estimated parameter sets $\theta_{1}$ and $\theta_{2}$. The null hypothesis of statistical indistinguishability of the two models is

$$
H_{0}: \operatorname{Pr}\left(k_{i}>0\right)=0.5 \quad \text { for all } i=1, \ldots, m,
$$

where

for observations $u_{i}, i=1, \ldots, m$.

$$
k_{i}=\log \left[\frac{c_{1}\left(u_{i} \mid \theta_{1}\right)}{c_{2}\left(u_{i} \mid \theta_{2}\right)}\right]
$$

Since under statistical equivalence of the two models the log likelihood ratios of the single observations are uniformly distributed around zero and in expectation $50 \%$ of the log likelihood ratios greater than zero, the test statistic

$$
B=\sum_{i=1}^{m} \mathbf{1}\left(0 \leq k_{i} \leq \infty\right)
$$

where $\mathbf{1}$ is the indicator function, has the binomial distribution with parameters $m$ and $p=0.5$. Model 1 is interpreted as statistically equivalent to model 2 if $B$ is not significantly different from the expected value $m p=m / 2$.

The likelihood-ratio based test proposed by V u ong (1989) [12 can be used for comparing non-nested models. For this let $c_{1}$ and $c_{2}$ be two densities of copulas with estimated parameter sets $\theta_{1}$ and $\theta_{2}$. We then compute the standardized sum $\nu$ of the $\log$ differences of their pointwise likelihoods

$$
k_{i}=\log \left[\frac{c_{1}\left(u_{i} \mid \theta_{1}\right)}{c_{2}\left(u_{i} \mid \theta_{2}\right)}\right]
$$

for observations $u_{i} \in[0,1], i=1, \ldots, m$, i.e., statistic

$$
\nu=\frac{1}{m} \frac{\sum_{i=1}^{m} k_{i}}{\sqrt{\sum_{i=1}^{m}\left(k_{i}-\bar{k}\right)^{2}}} \quad \text { with } \quad \bar{k}=\frac{1}{m} \sum_{i=1}^{m} k_{i} .
$$


V u ong (1989) 12 shows that $\nu$ is asymptotically standard normal. According to the null-hypothesis

$$
H_{0}: E\left[k_{i}\right]=0 \quad \text { for all } i=1, \ldots, m,
$$

we hence prefer model 1 to model 2 at level $\alpha$ if

$$
\nu>\Phi^{-1}(1-\alpha / 2),
$$

where $\Phi^{-1}$ denotes the inverse of the standard normal distribution function. If

$$
\nu<-\Phi^{-1}(1-\alpha / 2),
$$

we choose model 2. If, however,

$$
|\nu| \leq \Phi^{-1}(1-\alpha / 2),
$$

no decision among the models is possible.

\section{Modeling results}

All calculations were done in R [9] with the help of packages [7] and [10].

In package [10] several bivariate copula families are included for bivariate analysis as well as for multivariate analysis. It provides elliptical (Gaussian and Student-t) as well as Archimedean (Clayton, Gumbel, Frank, Joe, BB1, BB6, BB7 and BB8) copulas. For any copula families rotated versions

$$
\begin{gathered}
C_{90}\left(x_{1}, x_{2}\right)=x_{2}-C\left(1-x_{1}, x_{2}\right), \\
C_{180}\left(x_{1}, x_{2}\right)=x_{1}+x_{2}-1+C\left(1-x_{1}, 1-x_{2}\right) \quad \text { survival copula, } \\
C_{270}\left(x_{1}, x_{2}\right)=x_{1}-C\left(x_{1}, 1-x_{2}\right),
\end{gathered}
$$

are included to cover negative dependence, too. For more and detailed information about copula families see [8].

The Vuong as well as the Clarke test compare two models against each other allow for a decision among several models. In Belgorodski [1 this is used for bivariate copula selection. It compares a model 0 to all other possible models under consideration. If model 0 is favored over another model, a score of $+\mathbf{1}$ is assigned and similarly a score of $\mathbf{- 1}$ if the other model is determined to be superior. No score is assigned, if the respective test cannot discriminate between two models.

The models compared here are bivariate parametric copulas and we would like to determine which family fits the data better than the other families. The family with the highest score should be selected. For more and detailed information about the goodness-of-fit test see B elgorodski [1]. 


\section{J. KOMORNÍK - M. KOMORNÍKOVÁ - T. BACIGÁL - C. NGUYEN}

We can see the graphs of considered triple of time series in the Figure 1 We see that the Merrill Lynch US Corporate Bond Index (ML) mostly leads the remaining two in the considered triple (with deeper losses in the crisis period).

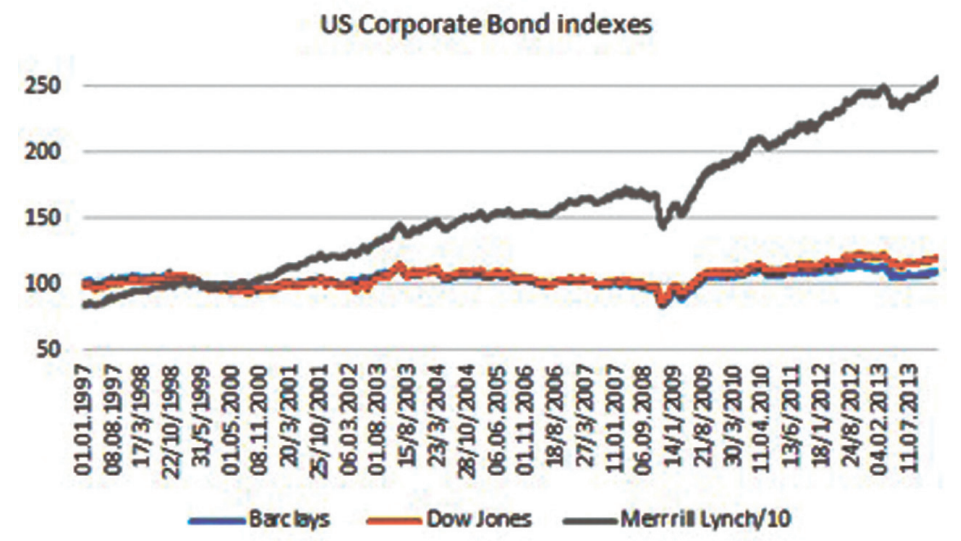

Figure 1. US Corporate Bond indices.

Before further analyses, we filtered all considered time series by ARMA-GARCH filters [4. Results of the introductory standard analysis of the residuals are presented in Table 1 and Figure 2, We see that the values of the Kendall's correlation coefficients are fairly high (contained in the interval [0.825, 0.836]) for all 3 considered couples of residuals. Their strong dependencies are illustrated in Figure 2,

TABLE 1. Values of the Kendall's correlation coefficient for all couples of the (filtered) returns of US CBI.

\begin{tabular}{|l|c|c|c|}
\hline & bar & $\mathrm{dj}$ & $\mathrm{ml}$ \\
\hline bar & 1 & 0.825 & 0.826 \\
$\mathrm{dj}$ & 0.825 & 1 & 0.836 \\
$\mathrm{ml}$ & 0.826 & 0.836 & 1 \\
\hline
\end{tabular}
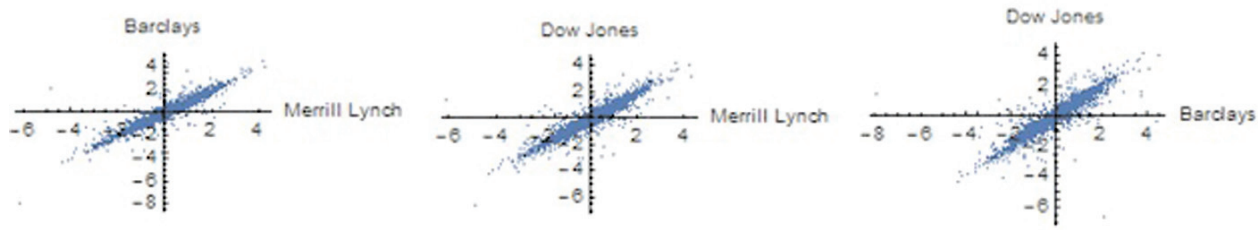

FIGURE 2. Scatter plot for all couples of the (filtered) returns of US CBI. 
We prolonged our analyses by examining developments of the Kendalls correlations. We have chosen annuals frequency of calculations of Kendalls correlation coefficients over the intervals of 24 months overlapping by 12 months with the intervals for calculation of the neighboring values of Kendalls correlation coefficient. Altogether, we have calculated a sequence of 17 such values. The last of them was calculated from the interval of 17 months. We can see (Figure 3) that all three correlation coefficients exhibit extremely parallel development and their values are contained in the interval [0.7, 0.95] of high values and by far do not approach the rejection limits for tests of their zero value (that equals $0.029)$.

\section{Kendall's tau}

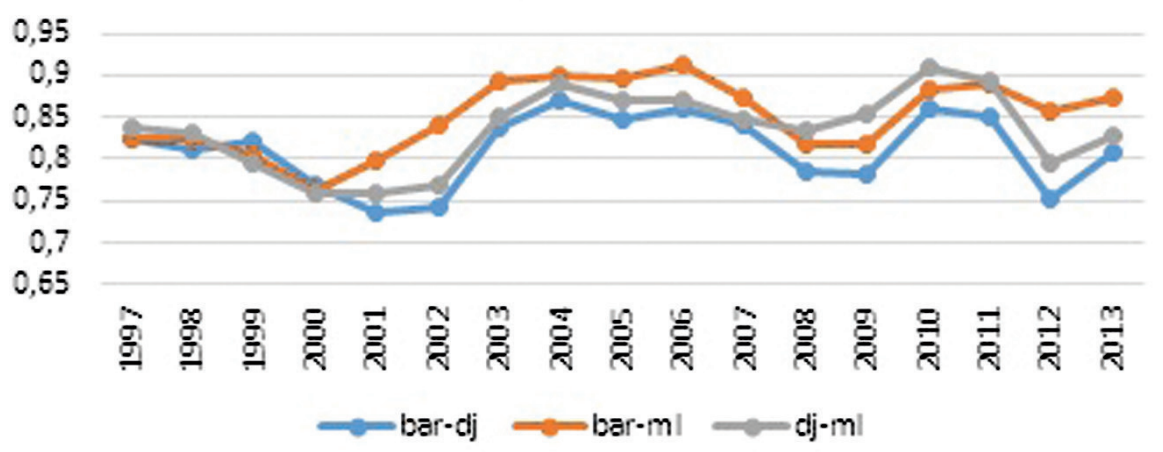

Figure 3. Evolution of Kendall's $\tau$ for all couples of the (filtered) returns of US CBI.

TABle 2. GoF test for 3D copulas, based on the Cramer-von-Mises method.

\begin{tabular}{|l|c|c|c|c|}
\hline Copula & par $_{1}$ & par $_{2}$ & par $_{3}$ & CvM \\
\hline Clayton & 5.847 & $\mathrm{x}$ & $\mathrm{x}$ & 5.236 \\
Frank & 21.470 & $\mathrm{x}$ & $\mathrm{x}$ & 0.441 \\
Gumbel & 5.221 & $\mathrm{x}$ & $\mathrm{x}$ & 0.772 \\
Gauss & 0.994 & $\mathrm{x}$ & $\mathrm{x}$ & 0.801 \\
Gauss1 & 0.928 & 0.957 & 0.947 & 0.765 \\
t-copula & 0.973 & $\mathrm{x}$ & $\mathrm{x}$ & 0.088 \\
t-copula1 & 0.967 & 0.977 & 0.973 & 0.082 \\
\hline
\end{tabular}

Next we calculated global copula models for the triple of considered series (see Table 2). We see that (among the considered copulas) the most competitive models (by far) provide Student class copulas, where one with 3 parameters 


\section{J. KOMORNÍK - M. KOMORNÍKOVÁ - T. BACIGÁL - C. NGUYEN}

(that have very similar values) is closely followed by the best one-parameter Student class model (with the parameter that is also close to the mentioned 3 parameters of the best 3-parametric model). The best copula with respect to Cramer-von-Mises (CvM) test statistic is the trivariate $t$-copula with test statistic $S_{C M}=0.082$ and parameters: and The best one-parameter Student class copula (with Cramer-von-Mises test statistic $S_{C M}=0.088$ ) has parameters: $\rho=0.973, \lambda_{L}=\lambda_{U}=0.804$ and degrees of freedom $d f=2$. A very low value of the degree of freedom indicate heavy tails that also correspond to fairly high values of the coefficients of tail dependence. Among the bivariate copula models the best ranking according to the Vuong test was achieved (for all three considered couples) by the models in the Student class quite closely followed by the models in the $B B 1_{180}$ class. According to the scoring based on the Clarke test, the results are slightly different. The models from the Student class are the best for the couple bar \& dj and dj \& ml, while they share the best rating with the $B B 1_{180}$ for the couple bar \& $\mathrm{ml}$ (see Table 3. Table 4 and Table 51). The optimal bivariate Student class copulas have 2 degrees of freedom for all three couples. Their contour plots are presented in Figure 4.

TABLE 3. Results for Vuong-Clarke scoring test for couple bar \& dj.

\begin{tabular}{|l|c|c|c|c|c|c|}
\hline Copula & $\theta_{1}$ & $\theta_{2}$ & $\lambda_{L}$ & $\lambda_{U}$ & Vuong & Clarke \\
\hline Normal & 0.928 & $\mathrm{x}$ & 0 & 0 & -5 & 0 \\
t Copula & 0.965 & $\mathrm{x}$ & 0.777 & 0.777 & 16 & 15 \\
Clayton & 5.507 & $\mathrm{x}$ & 0.802 & 0 & -8 & -4 \\
Gumbel & 4.897 & $\mathrm{x}$ & 0 & 0.848 & 7 & 6 \\
Frank & 20.602 & $\mathrm{x}$ & 0 & 0 & 8 & 14 \\
Joe & 5.944 & $\mathrm{x}$ & 0 & 0.876 & -7 & -8 \\
BB1 & 0.736 & 3.619 & 0.771 & 0.789 & 12 & 9 \\
BB6 & 1.001 & 4.717 & 0 & 0.842 & -15 & -15 \\
BB7 & 4.312 & 3.761 & 0.832 & 0.826 & 2 & 2 \\
BB8 & 5.000 & 0.963 & 0 & 0 & -4 & -12 \\
rClayton & 4.908 & $\mathrm{x}$ & 0 & 0.868 & -10 & -10 \\
rGumbel & 4.855 & $\mathrm{x}$ & 0.846 & 0 & 8 & 9 \\
rJoe & 6.044 & $\mathrm{x}$ & 0.878 & 0 & -6 & -2 \\
rBB1 & 0.527 & 3.950 & 0.808 & 0.006 & 14 & 13 \\
rBB6 & 1.001 & 4.852 & 0.847 & 0 & -15 & -15 \\
rBB7 & 4.704 & 3.286 & 0.841 & 0.810 & 5 & 4 \\
rBB8 & 6.000 & 0.970 & 0 & 0 & -2 & -6 \\
\hline
\end{tabular}


MULTIDIMENSIONAL COPULA MODELS FOR PARALLEL DEVELOPMENT...
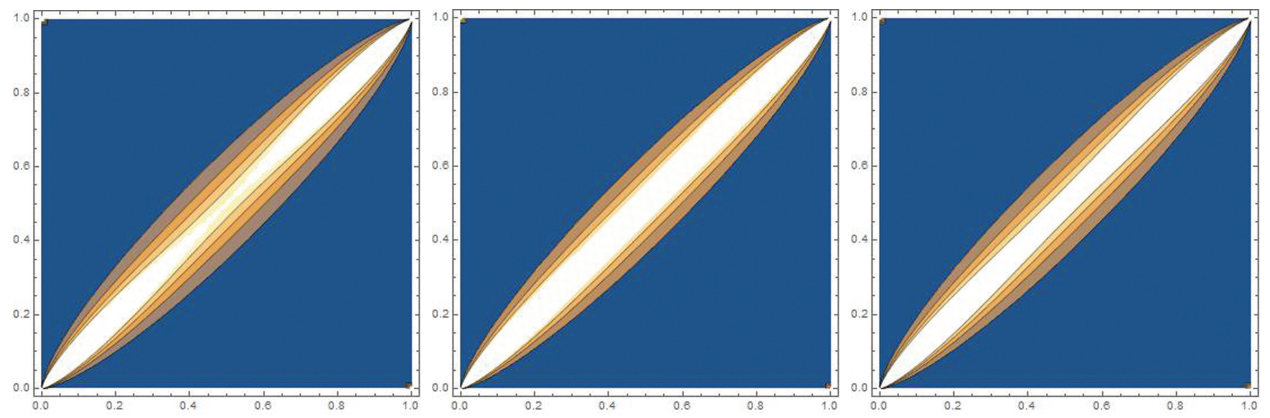

Figure 4. Contour plot of densities of t-copulas for couples bar \& dj (left), bar \& ml (middle) and dj \& ml (right).

TABLE 4. Results for Vuong-Clarke scoring test for couple bar \& ml.

\begin{tabular}{|l|c|c|c|c|c|c|}
\hline Copula & $\theta_{1}$ & $\theta_{2}$ & $\lambda_{L}$ & $\lambda_{U}$ & Vuong & Clarke \\
\hline Normal & 0.957 & $\mathrm{x}$ & 0 & 0 & -7 & 0 \\
t Copula & 0.976 & $\mathrm{x}$ & 0.815 & 0.815 & 16 & 14 \\
Clayton & 7.455 & $\mathrm{x}$ & 0.911 & 0 & -7 & -4 \\
Gumbel & 6.069 & $\mathrm{x}$ & 0 & 0.879 & 4 & 4 \\
Frank & 25.580 & $\mathrm{x}$ & 0 & 0 & 2 & 11 \\
Joe & 7.380 & $\mathrm{x}$ & 0 & 0.901 & -13 & -11 \\
BB1 & 0.987 & 4.205 & 0.846 & 0.821 & 12 & 10 \\
BB6 & 1.001 & 5.902 & 0 & 0.876 & 4 & 2 \\
BB7 & 5.000 & 5.949 & 0.890 & 0.851 & 0 & -5 \\
BB8 & 6.000 & 0.985 & 0 & 0 & -14 & -16 \\
rClayton & 6.374 & $\mathrm{x}$ & 0 & 0.897 & -15 & -12 \\
rGumbel & 6.240 & $\mathrm{x}$ & 0.882 & 0 & 9 & 11 \\
rJoe & 8.032 & $\mathrm{x}$ & 0.910 & 0 & -5 & -3 \\
rBB1 & 0.483 & 5.143 & 0.856 & 0.001 & 14 & 14 \\
rBB6 & 1.001 & 6.000 & 0.878 & 0 & 9 & 6 \\
rBB7 & 5.000 & 4.563 & 0.851 & 0.859 & 0 & -8 \\
rBB8 & 6.000 & 0.993 & 0 & 0 & -9 & -13 \\
\hline
\end{tabular}

Based on the above mentioned results (together with the dominance of the Student class among the 3 -dimensional models that is also providing a supporting argument for optimality of the Student class bivariate copulas for all 3 pairs of the considered indices) we can conclude that the investigated triple of investment returns exhibit (pairwise) high values of tail dependencies. 
TABLE 5. Results for Vuong-Clarke scoring test for couple dj \& ml.

\begin{tabular}{|l|c|c|c|c|c|c|}
\hline Copula & $\theta_{1}$ & $\theta_{2}$ & $\lambda_{L}$ & $\lambda_{U}$ & Vuong & Clarke \\
\hline Normal & 0.947 & $\mathrm{x}$ & 0 & 0 & -4 & 0 \\
t Copula & 0.972 & $\mathrm{x}$ & 0.788 & 0.788 & 16 & 15 \\
Clayton & 6.370 & $\mathrm{x}$ & 0.867 & 0 & -12 & -8 \\
Gumbel & 5.626 & $\mathrm{x}$ & 0 & 0.869 & 8 & 6 \\
Frank & 23.077 & $\mathrm{x}$ & 0 & 0 & 0 & 13 \\
Joe & 6.950 & $\mathrm{x}$ & 0 & 0.895 & -10 & -9 \\
BB1 & 0.987 & 4.250 & 0.846 & 0.821 & 13 & 11 \\
BB6 & 1.001 & 5.902 & 0 & 0.876 & 6 & 4 \\
BB7 & 5.000 & 5.949 & 0.890 & 0.851 & 0 & -3 \\
BB8 & 6.000 & 0.985 & 0 & 0 & -11 & -15 \\
rClayton & 6.374 & $\mathrm{x}$ & 0 & 0.897 & -12 & -10 \\
rGumbel & 6.240 & $\mathrm{x}$ & 0.882 & 0 & 8 & 6 \\
rJoe & 8.032 & $\mathrm{x}$ & 0.910 & 0 & -10 & -7 \\
rBB1 & 0.483 & 5.143 & 0.856 & 0.001 & 13 & 13 \\
rBB6 & 1.001 & 6.000 & 0.878 & 0 & 6 & 4 \\
rBB7 & 5.000 & 4.563 & 0.851 & 0.859 & 0 & -5 \\
rBB8 & 6.000 & 0.993 & 0 & 0 & -11 & -15 \\
\hline
\end{tabular}

We also calculated locally best models on the system of 2-years' intervals mentioned above. Development of their rating scores based both on Vuong and Clarke tests for all considered couples are presented in Figures 5, 6, 0, A clear local dominance of the Student class models is demonstrated.
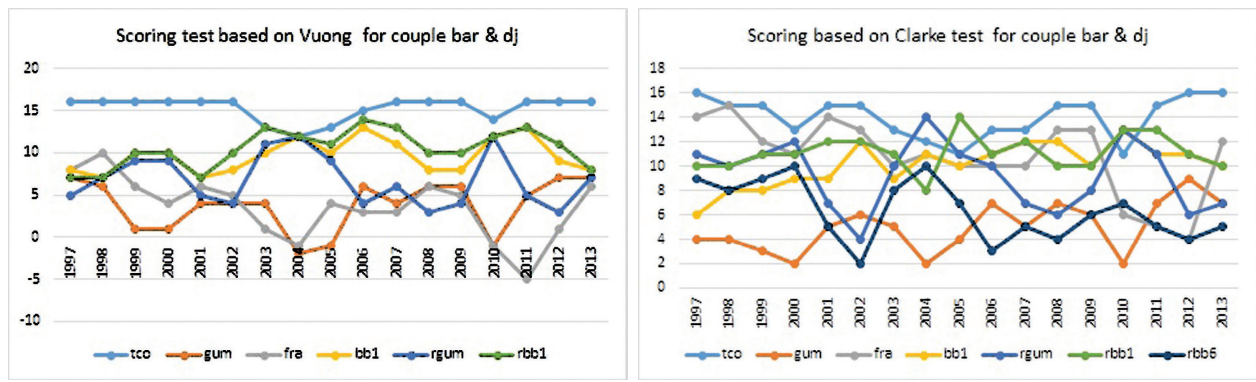

FiguRE 5. Evolution of scoring based on Vuong (left) and Clarke (right) tests for couple bar \& dj. 

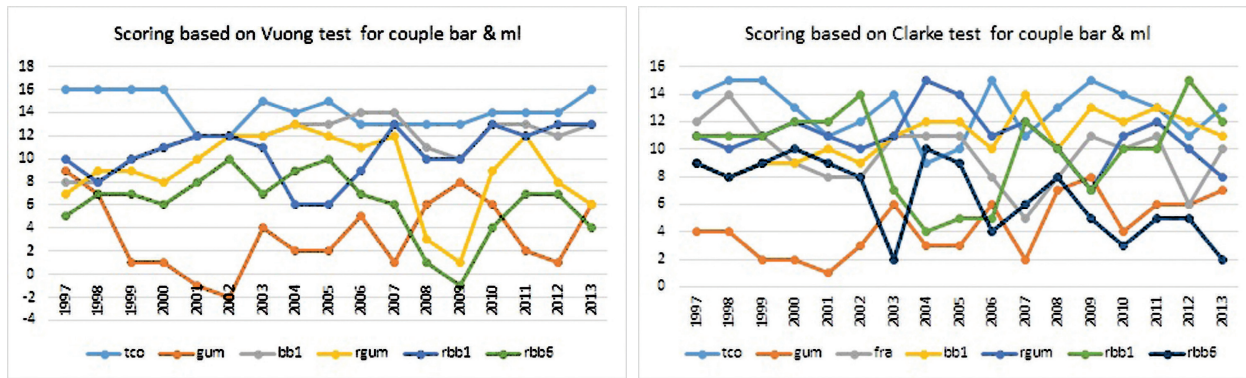

FiguRE 6. Evolution of scoring based on Vuong (left) and Clarke (right) tests for couple bar \& ml.
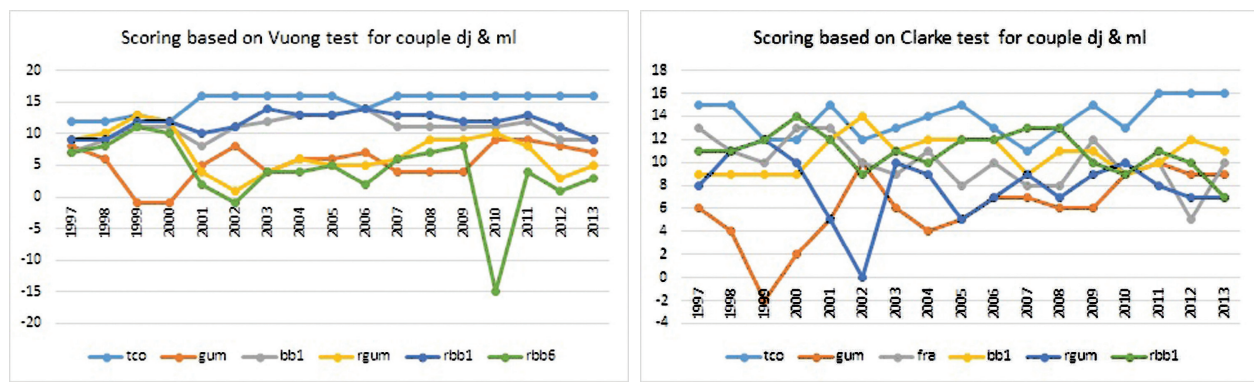

FiguRe 7. Evolution of scoring based on Vuong (left) and Clarke (right) tests for couple dj \& ml.

Figure 8 contains graphs of development of the parameters and tail dependence coefficients of the best Student local models.
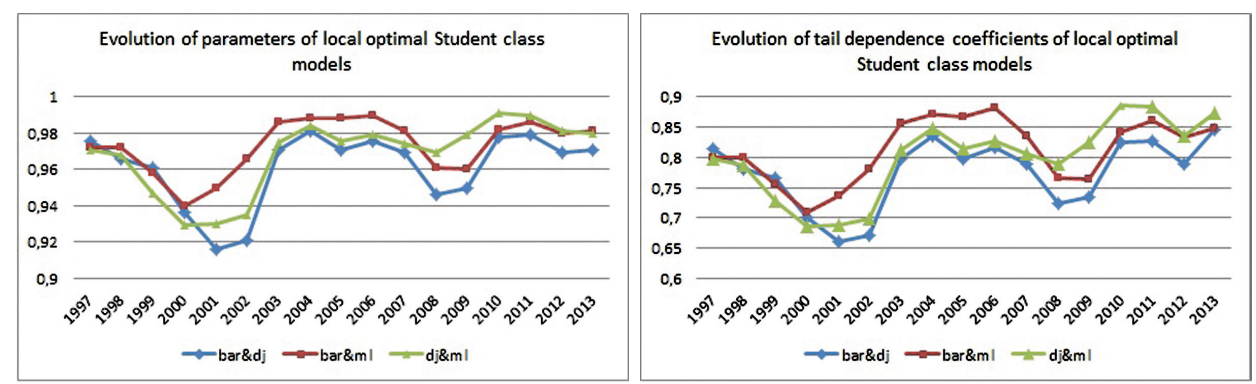

Figure 8. Evolution of parameters (left) and tail dependence coefficients (right) of local optimal Student class models. 


\section{J. KOMORNÍK - M. KOMORNÍKOVÁ - T. BACIGÁL - C. NGUYEN}

Finally, Figure 9 contains comparable graphs of the development of the parameters and tail dependence coefficients of the optimal one-parametric Student $3 \mathrm{D}$ models.

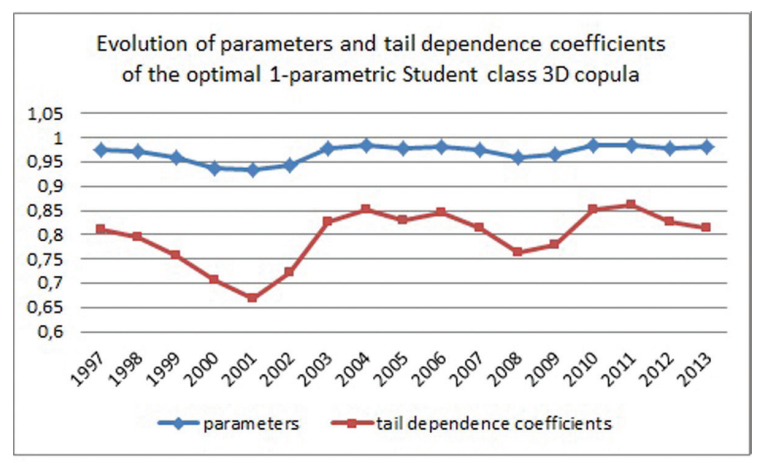

FiguRE 9. Evolution of parameters and tail dependence coefficients of the optimal 1-parametric Student class 3D copula.

\section{Concluding remarks}

We concluded that the returns of the considered bond indices have very high coefficients tail dependence and thus do not represent suitable components of well diversified portfolios. On the other hand, their optimal models are in the Student class with low degrees of freedom (only 2!) that implies high values of quantile functions, hence they could be good candidates for underlying assets of derivative instruments.

The results of our modeling are very impressive. The trends in development of the outcomes of the local models are surprisingly parallel. The values of pairwise correlations between the considered inputs as well as of the calculated coefficients of tail dependence of local optimal partial pairwise 2D copulas and one-parametric local 3D Student copulas exhibit extremely similar behavior.

\section{REFERENCES}

[1] BELGORODSKI, N.: Selecting Pair-Copula Families for Regular Vines with Application to the Multivariate Analysis of European Stock Market Indices. Diploma thesis, Technische Universitaet Muenchen, http://mediatum.ub.tum.de/?id=1079284

[2] CLARKE, K. A.: A simple distribution-free test for nonnested model selection, Political Analysis 15 (2007), 347-363. 


\section{MULTIDIMENSIONAL COPULA MODELS FOR PARALLEL DEVELOPMENT...}

[3] FERREIRA, M.: Nonparametric estimation of the Tail-dependence coefficient, REVSTAT 11 (2013), 1-16.

[4] FRAnSES, P. H.-DIJK, D.: Non-Linear Time Series Models in Empirical Finance. Cambridge University Press, Cambridge, 2000.

[5] GENEST, C.-RIVEST, L.-P.: Statistical inference procedures for bivariate Archimedean copulas, J. Amer. Statist. Assoc. 88 (1993), no. 423, 1034-1043.

[6] GENEST, C.-RÉMILLARD, B.-BEAUDOIN, D.: Goodness-of-fit tests for copulas: A review and a power study, Insur. Math. Econ. 44 (2009), 199-213.

[7] HOFERT, M.-KOJADINOVIC, I.-MAECHLER, M.-YAN, J.: Copula: Multivariate Dependence with Copulas. R package version 0.999-13, 2015. http://CRAN.R-project.org/package=copula

[8] NELSEN, R. B.: An Introduction to Copulas (2nd ed.), in: Springer Ser. Stat., Springer-Verlag, New York, 2006.

[9] R CORE TEAM.: R: A language and environment for statistical computing, R Foundation for Statistical Computing, Vienna, Austria, 2015. https://www.R-project.org/

[10] SCHEPSMEIER, U. ET AL.: VineCopula: statistical inference of vine copulas, R package version 1.6-1, 2015. http://CRAN.R-project.org/package=VineCopula

[11] SKLAR, A.: Fonctions de répartition a $n$ dimensions et leurs marges, Publ. Inst. Statist. Univ. Paris 8 (1959), 229-231.

[12] VUONG, Q. H.: Ratio tests for model selection and non-nested hypotheses, Econometrica 57 (1989), 307-333.

Received April 13, 2017
Jozef Komornik

Faculty of Management

Comenius University

Odbojárov 10

SK-831-04 Bratislava

SLOVAKIA

E-mail: Jozef.Komornik@fm.uniba.sk

Magdaléna Komorniková

Tomáš Bacigál

Faculty of Civil Engineering STU

Radlinského 11

SK-810-05 Bratislava

SLOVAKIA

E-mail: Magdalena.Komornikova@stuba.sk Tomas.Bacigal@stuba.sk

Cuong Nguyen

Faculty of Commerce

Lincoln University $N Z$

Canterbury

NEW ZEALAND

E-mail: Cuong.Nguyen@lincoln.ac.nz 